\title{
QUASI-HOMOLOGY AND UNIVERSAL COEFFICIENTS
}

\author{
by N. C. HSU
}

(Received 7 October, 1972)

In order to study an arbitrary sequence of modules and homomorphisms, we propose a definition of "homology" modules, or what we call quasi-homology modules, for such a sequence. Then we seek partial analogues of the universal coefficient theorems to make some propaganda for the notion.

1. Quasi-homology module. For a sequence

$$
C: \cdots \rightarrow C_{n+1} \stackrel{d_{n+1}}{\rightarrow} C_{n} \stackrel{d_{n}}{\rightarrow} C_{n-1} \rightarrow \cdots, \quad n \in \mathbf{Z},
$$

of arbitrary modules over a ring with an identity and arbitrary homomorphisms, we define the n-dimensional quasi-homology module $\mathscr{H}_{n}(C)$, the n-dimensional lower quasi-homology module $\underline{\mathscr{H}}_{n}(C)$ and the $n$-dimensional upper quasi-homology module $\overrightarrow{\mathscr{H}}_{n}(C)$ by

and

$$
\begin{aligned}
& \mathscr{H}_{n}(C)=\left(\operatorname{Ker} d_{n}+\operatorname{Im} d_{n+1}\right) /\left(\operatorname{Ker} d_{n} \cap \operatorname{Im} d_{n+1}\right), \\
& \mathscr{H}_{n}(C)=\operatorname{Ker} d_{n} /\left(\operatorname{Ker} d_{n} \cap \operatorname{Im} d_{n+1}\right)
\end{aligned}
$$

respectively.

$$
\mathscr{H}_{n}(C)=\operatorname{Im} d_{n+1} /\left(\operatorname{Ker} d_{n} \cap \operatorname{Im} d_{n+1}\right),
$$

If $C$ and $C^{\prime}$ are sequences of modules over the same ring, a homomorphism $f: C \rightarrow C^{\prime}$ is a family of homomorphisms $f_{n}: C_{n} \rightarrow C_{n}^{\prime}$, one for each $n$, such that $d_{n}^{\prime} f_{n}=f_{n-1} d_{n}$. The mapping

defined by

$$
\mathscr{H}_{n}(f): \quad \mathscr{H}_{n}(C) \rightarrow \mathscr{H}_{n}\left(C^{\prime}\right)
$$

$$
\mathscr{H}_{n}(f): \quad c+\left(\operatorname{Ker} d_{n} \cap \operatorname{Im} d_{n+1}\right) \mapsto f_{n}(c)+\left(\operatorname{Ker} d_{n}^{\prime} \cap \operatorname{Im} d_{n+1}^{\prime}\right)
$$

is a homomorphism. The same rule defines homomorphisms

and

$$
\underline{\mathscr{H}}_{n}(f): \underline{\mathscr{H}}_{n}(C) \rightarrow \underline{\mathscr{H}}_{n}\left(C^{\prime}\right)
$$

$$
\overline{\mathscr{H}}_{n}(f): \quad \overline{\mathscr{H}}_{n}(C) \rightarrow \overline{\mathscr{H}}_{n}\left(C^{\prime}\right) \text {. }
$$

With these definitions, $\mathscr{H}_{n}, \underline{\mathscr{H}}_{n}$ and $\overrightarrow{\mathscr{H}}_{n}$ are covariant functors on the category of sequences of modules to the category of modules for each $n$.

A homotopy $s$ between two homomorphisms $f, g: C \rightarrow C^{\prime}$ is a family of homomorphisms $s_{n}: C_{n} \rightarrow C_{n+1}^{\prime}$, one for each $n$, such that

$$
\begin{aligned}
d_{n+1}^{\prime} s_{n}+s_{n-1} d_{n} & =f_{n}-g_{n}, \\
s_{n-1} d_{n} d_{n+1} & =0
\end{aligned}
$$


and

$$
d_{n}^{\prime} d_{n+1}^{\prime} s_{n}=0
$$

for each $n$. The statements expected of homotopy such as Theorem 2.1, Corollary 2.2 and Proposition 2.3 in $[1$, p. 40] can readily be ascertained.

A sequence $C$ is said to be exact, demi-exact or semi-exact at $C_{n}$ according as $\mathscr{H}_{n}(C)=$ $0, \mathscr{H}_{n}(C)=0$ or $\overline{\mathscr{H}}_{n}(C)=0$. When a sequence $C$ is semi-exact at $C_{n}$, we have $\mathscr{H}_{n}(C)=$ $\mathscr{\mathscr { H }}_{n}(C)=H_{n}(C)$, where $H_{n}(C)$ is the usual $n$-dimensional homology module of $C$. A sequence $\bar{C}$ is said to be exact, demi-exact or semi-exact according as the sequence $C$ is exact, demi-exact or semi-exact at $C_{n}$ for every $n$. Semi-exact sequences are the ones that have been most intensively studied until now. All sequences consisting entirely of epimorphisms, and all sequences consisting entirely of monomorphisms, are demi-exact.

\section{Lower and upper quasi-homology modules.}

PROPOSITION 2.1. For any sequence $C$ and for any integer $n$, we have

$$
\mathscr{H}_{n}(C)=\underline{\mathscr{H}}_{n}(C) \oplus \overline{\mathscr{H}}_{n}(C) \text {. }
$$

We use the sign \to mean that no proof is given or to indicate the end of the proof.

From a sequence

$$
C: \cdots \rightarrow C_{n+1} \stackrel{d_{n+1}}{\rightarrow} C_{n} \stackrel{d_{n}}{\rightarrow} C_{n-1} \rightarrow \cdots,
$$

we form a new sequence

$$
d^{-2} C: \cdots \rightarrow \operatorname{Ker} d_{n} d_{n+1} \stackrel{d_{n+1}^{-2}}{\rightarrow} \operatorname{Ker} d_{n-1} d_{n} \stackrel{d_{n}^{-2}}{\rightarrow} \operatorname{Ker} d_{n-2} d_{n-1} \rightarrow \cdots,
$$

where $d_{n}^{-2}(c)=d_{n}(c)$ for all $c \in \operatorname{Ker} d_{n-1} d_{n} \subset C_{n}$ for each $n$.

Proposition 2.2. For any sequence $C$, the sequence $d^{-2} C$ is semi-exact and we have

$$
\mathscr{H}_{n}(C)=H_{n}\left(d^{-2} C\right)
$$

for each integer $n$.

Proof. $\operatorname{Im} d_{n+1}^{-2}=\operatorname{Ker} d_{n} \cap \operatorname{Im} d_{n+1} \subset \operatorname{Ker} d_{n}=\operatorname{Ker} d_{n}^{-2}$, and

$$
\begin{aligned}
\mathscr{\mathscr { H }}_{n}(C) & =\operatorname{Ker} d_{n} /\left(\operatorname{Ker} d_{n} \cap \operatorname{Im} d_{n+1}\right) \\
& =\operatorname{Ker} d_{n}^{-2} / \operatorname{Im} d_{n+1}^{-2}=H_{n}\left(d^{-2} C\right) .
\end{aligned}
$$

Proposition 2.3. For any sequence $C$ and for any integer n, we have isomorphisms

under the mappings

$$
C_{n+1} / \operatorname{Ker} d_{n} d_{n+1} \nrightarrow \overline{\mathscr{H}}_{n}(C) \nrightarrow \operatorname{Im} d_{n} d_{n+1}
$$

$$
\begin{gathered}
i_{1}(C) \\
c+\operatorname{Ker} d_{n} d_{n+1} \stackrel{i_{2}(C)}{\mapsto} d_{n+1}(c)+\left(\operatorname{Ker} d_{n} \cap \operatorname{Im} d_{n+1}\right) \stackrel{\leftrightarrow}{\mapsto} d_{n} d_{n+1}(c) .
\end{gathered}
$$


3. Universal coefficients for quasi-homology. Throughout the section, let

$$
C: \cdots \rightarrow C_{n+1} \stackrel{d_{n+1}}{\rightarrow} C_{n} \rightarrow C_{n-1} \rightarrow \cdots
$$

be a sequence of modules over a commutative ring with an identity and let $G$ be a module over the same ring. We consider

$$
C \otimes G: \cdots \rightarrow C_{n+1} \otimes G \stackrel{d_{n+1}^{\hat{n}} \rightarrow}{\rightarrow} C_{n} \otimes G \stackrel{d_{n}^{\wedge}}{\rightarrow} C_{n-1} \otimes G \rightarrow \cdots,
$$

where $d_{n}^{\wedge}=d_{n} \otimes 1_{G}$. We are interested in the $n$-dimensional quasi-homology module $\mathscr{H}_{n}(C \otimes G)$ of $C \otimes G$. By Proposition 2.1, we have the decompositions

and

$$
\mathscr{H}_{n}(C) \otimes G=\left(\underline{\mathscr{H}}_{n}(C) \otimes G\right) \oplus\left(\overline{\mathscr{H}}_{n}(C) \otimes G\right)
$$

$$
\mathscr{H}_{n}(C \otimes G)=\underline{\mathscr{H}}_{n}(C \otimes G) \oplus \overline{\mathscr{H}}_{n}(C \otimes G) .
$$

Proposition 3.1. For any sequence $C$, for any module $G$ over the same ring and for any integer $n$, the mapping

$$
\alpha_{n}: \quad \mathscr{H}_{n}(C) \otimes G \rightarrow \mathscr{H}_{n}(C \otimes G)
$$

defined as follows is a homomorphism:

where

$$
\alpha_{n}: \quad\left(c+\left(\operatorname{Ker} d_{n} \cap \operatorname{Im} d_{n+1}\right)\right) \otimes g \mapsto(c \otimes g)+\left(\operatorname{Ker} d_{n}^{\wedge} \cap \operatorname{Im} d_{n+1}^{\wedge}\right),
$$

If

$$
c \in \operatorname{Ker} d_{n}+\operatorname{Im} d_{n+1} \subset C_{n}, g \in G \text { and } c \otimes g \in \operatorname{Ker} d_{n}^{\wedge}+\operatorname{Im} d_{n+1}^{\wedge} \subset C_{n} \otimes G \text {. }
$$

and

$$
\underline{\alpha}_{n}=\alpha_{n} \mid \underline{\mathscr{H}}_{n}(C) \otimes G
$$

then

$$
\bar{\alpha}_{n}=\alpha_{n} \mid \overline{\mathscr{H}}_{n}(C) \otimes G
$$

and

$$
\underline{\alpha}_{n}: \quad \underline{\mathscr{H}}_{n}(C) \otimes G \rightarrow \underline{\mathscr{H}}_{n}(C \otimes G)
$$

$$
\bar{\alpha}_{n}: \quad \mathscr{\mathscr { H }}_{n}(C) \otimes G \rightarrow \overline{\mathscr{H}}_{n}(C \otimes G) .
$$

PRoposition 3.2. For any sequence $C$, for any module $G$ over the same ring and for any integer $n$, if there exist isomorphisms $f_{n+1}, f_{n}$ and $f_{n-1}$ making

$$
\begin{array}{ccccc}
\operatorname{Ker} d_{n}^{\wedge} d_{n+1}^{\wedge} & \stackrel{d_{n+1}^{\wedge-2}}{\rightarrow} \operatorname{Ker} d_{n-1}^{\wedge} d_{n}^{\wedge} & \multicolumn{1}{c}{d_{n}^{\wedge-2}} \\
\downarrow f_{n+1} & & \downarrow & & \operatorname{Ker} d_{n-2}^{\wedge} d_{n-1}^{\wedge} \\
& d_{n+1}^{-2 \wedge} & \downarrow f_{n} & & \downarrow f_{n-1}
\end{array}
$$

commutative, then

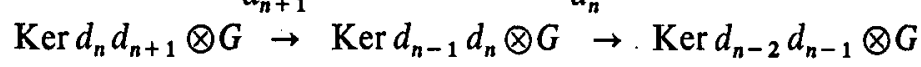

and

$$
\underline{\mathscr{H}}_{n}(C) \otimes G=H_{n}\left(d^{-2} C\right) \otimes G
$$

$$
\underline{\mathscr{H}}_{n}(C \otimes G)=H_{n}\left(d^{-2} C \otimes G\right)
$$


Proof. Under the hypothesis, the two semi-exact sequences $d^{\wedge-2}(C \otimes G)$ and $d^{-2} C \otimes G$ have the same $n$-dimensional homology module and Proposition 2.2 yields

$$
\underline{\mathscr{H}}_{n}(C \otimes G)=H_{n}\left(d^{\wedge-2}(C \otimes G)\right)=H_{n}\left(d^{-2} C \otimes G\right) \text {. }
$$

Needless to say, if $C$ is semi-exact, then the hypothesis of Proposition 3.2 is obviously satisfied.

Proposition 3.3. For any sequence $C$, for any free module $G$ over the same ring and for any integer $n$, the hypothesis of Proposition 3.2 is satisfied.

Proof. If $G$ is free, we have a monomorphism

$$
j \otimes 1_{G}: \quad \operatorname{Ker} d_{n-1} d_{n} \otimes G \rightarrow C_{n} \otimes G,
$$

where $j$ is the injection, i.e. the inclusion, of $\operatorname{Ker} d_{n-1} d_{n}$ into $C_{n}$. Since $G$ is free, we have

$$
\operatorname{Im}\left(j \otimes 1_{G}\right)=\operatorname{Ker} d_{n-1}^{\wedge} d_{n}^{\wedge},
$$

and therefore $j \otimes 1_{G}$ induces an isomorphism of $\operatorname{Ker} d_{n-1} d_{n} \otimes G$ onto $\operatorname{Ker} d_{n-1}^{\wedge} d_{n}^{\wedge}$. Let $f_{n}$ be the inverse of this isomorphism. The isomorphisms $f_{n+1}, f_{n}$ and $f_{n-1}$ clearly satisfy the hypothesis of Proposition 3.2.

Proposition 3.2 means that, under the hypothesis stated, the study of

$$
\underline{\alpha}_{n}: \quad \underline{H}_{n}(C) \otimes G \rightarrow \underline{H}_{n}(C \otimes G)
$$

is reduced to the study of the homomorphism

$$
H_{n}\left(d^{-2} C\right) \otimes G \rightarrow H_{n}\left(d^{-2} C \otimes G\right)
$$

in the usual universal coefficient theorem for homology [1, p. 171]. In particular, we record

Proposition 3.4. For any sequence $C$ of free modules over a principal ideal domain, for any projective module $G$ over the same domain and for any integer $n$, if the hypothesis of Proposition 3.2 is satisfied, then

defined by

$$
\underline{\alpha}_{n}: \quad \underline{\mathscr{H}}_{n}(C) \otimes G \rightarrow \underline{\mathscr{H}}_{n}(C \otimes G)
$$

$$
\underline{\alpha}_{n}: \quad\left(c+\left(\operatorname{Ker} d_{n} \cap \operatorname{Im} d_{n+1}\right)\right) \otimes g \mapsto(c \otimes g)+\left(\operatorname{Ker} d_{n}^{\wedge} \cap \operatorname{Im} d_{n+1}^{\wedge}\right)
$$

is an isomorphism.

COROLlARY 3.5. For any sequence $C$ of vector spaces over a field, for any vector space $G$ over the same field and for any integer $n$,

defined by

$$
\underline{\alpha}_{n}: \quad \underline{\mathscr{H}}_{n}(C) \otimes G \rightarrow \underline{\mathscr{H}}_{n}(C \otimes G)
$$

$$
\underline{\alpha}_{n}: \quad\left(c+\left(\operatorname{Ker} d_{n} \cap \operatorname{Im} d_{n+1}\right)\right) \otimes g \mapsto(c \otimes g)+\left(\operatorname{Ker} d_{n}^{\wedge} \cap \operatorname{Im} d_{n+1}^{\wedge}\right)
$$

is an isomorphism.

Proof. Combine Proposition 3.3 and Proposition 3.4. 
The usual universal coefficient theorem for homology states among other things that, under certain circumstances, the homomorphism similar to our $\underline{\alpha}_{n}$ is a monomorphism. It is interesting to note that $\bar{\alpha}_{n}$ is an epimorphism. This is part of the following theorem.

THEOREM 3.6. Let $C$ be a sequence of modules and let $G$ be a module over the same ring. For any integer $n$, let

be a mapping defined by

$$
\bar{\alpha}_{n}: \quad \overline{\mathscr{H}}_{n}(C) \otimes G \rightarrow \overline{\mathscr{H}}_{n}(C \otimes G)
$$

Then

$$
\bar{\alpha}_{n}: \quad\left(c+\left(\operatorname{Ker} d_{n} \cap \operatorname{Im} d_{n+1}\right)\right) \otimes g \mapsto(c \otimes g)+\left(\operatorname{Ker} d_{n}^{\wedge} \cap \operatorname{Im} d_{n+1}^{\wedge}\right) .
$$

(1) $\bar{\alpha}_{n}$ is an epimorphism.

(2) If $C_{n-1}$ is projective, then

$$
0 \rightarrow \operatorname{Tor}\left(C_{n-1} / \overline{\mathscr{H}}_{n}(C), G\right) \rightarrow \overline{\mathscr{H}}_{n}(C) \otimes G \stackrel{\bar{\alpha}_{n}}{\rightarrow} \overline{\mathscr{H}}_{n}(C \otimes G) \rightarrow 0
$$

is exact.

(3) If $G$ is projective, then (regardless of whether $C_{n-1}$ is projective or not) $\bar{\alpha}_{n}$ is an isomorphism.

Proof. Let $j_{1}$ be the injection, i.e. the inclusion, of $\operatorname{Im} d_{n} d_{n+1}$ into $C_{n-1}$ and recall that $i_{2}(C)$ and $i_{2}(C \otimes G)$ are the isomorphisms

and

$$
i_{2}(C): \quad \overline{\mathscr{H}}_{n}(C) \nrightarrow \operatorname{Im} d_{n} d_{n+1}
$$

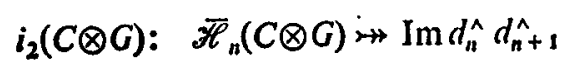

given in Proposition 2.3. From a short exact sequence

$$
0 \rightarrow \overline{\mathscr{H}}_{n}(C) \stackrel{j_{1} i_{2}(C)}{\rightarrow} C_{n-1} \rightarrow C_{n-1} / \overline{\mathscr{H}}_{n}(C) \rightarrow 0,
$$

we obtain its fundamental exact sequence

$$
\begin{gathered}
\cdots \rightarrow \operatorname{Tor}\left(C_{n-1}, G\right) \rightarrow \operatorname{Tor}\left(C_{n-1} / \overline{\mathscr{H}}_{n}(C), G\right) \\
\rightarrow \mathscr{H}_{n}(C) \otimes G \stackrel{j_{1}^{\wedge} i_{2}(C)^{\wedge}}{\rightarrow} C_{n-1} \otimes G \rightarrow\left(C_{n-1} / \overline{\mathscr{H}}_{n}(C)\right) \otimes G \rightarrow 0 .
\end{gathered}
$$

A quick computation tells us that

$$
\operatorname{Im} j_{1}^{\wedge} i_{2}(C)^{\wedge}=\operatorname{Im} d_{n}^{\wedge} d_{n+1}^{\wedge} .
$$

In view of the commutative diagram

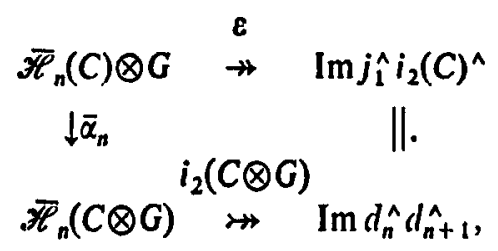


where $\varepsilon$ is the epimorphism induced by $j_{1}^{\wedge} i_{2}(C)^{\wedge}$ by restricting its codomain, the fundamental exact sequence yields an exact sequence

$$
\begin{aligned}
\cdots \rightarrow & \text { Tor }\left(C_{n-1}, G\right) \rightarrow \operatorname{Tor}\left(C_{n-1} / \overline{\mathscr{H}}_{n}(C), G\right) \\
& \rightarrow \overline{\mathscr{H}}_{n}(C) \otimes G \rightarrow \bar{\alpha}_{n} \overline{\mathscr{H}}_{n}(C \otimes G) \rightarrow 0 .
\end{aligned}
$$

The conclusions follow from this immediately.

CoROLlaRY 3.7. Let $C$ be a sequence of vector spaces over a field and let $G$ be a vector space over the same field. For any integer $n$, the mapping

defined by

$$
\alpha_{n}: \quad \mathscr{H}_{n}(C) \otimes G \rightarrow \mathscr{H}_{n}(C \otimes G)
$$

$$
\alpha_{n}: \quad\left(c+\left(\operatorname{Ker} d_{n} \cap \operatorname{Im} d_{n+1}\right)\right) \otimes g \mapsto(c \otimes g)+\left(\operatorname{Ker} d_{n}^{\wedge} \cap \operatorname{Im} d_{n+1}^{\wedge}\right)
$$

is an isomorphism.

Proof. Combine Corollary 3.5 and (3) of Theorem 3.6.

4. Universal coefficients for quasi-cohomology. Throughout the section, let

$$
C: \cdots \rightarrow C_{n+1} \stackrel{d_{n+1}}{\rightarrow} C_{n} \rightarrow C_{n-1} \rightarrow \cdots
$$

be a sequence of modules over a commutative ring with an identity and let $G$ be a module over the same ring. We consider

$$
\operatorname{Hom}(C, G): \cdots \leftarrow \operatorname{Hom}\left(C_{n+1}, G\right) \stackrel{d_{n+1}^{*}}{\leftarrow} \operatorname{Hom}\left(C_{n}, G\right) \stackrel{d_{n}^{*}}{\leftarrow \operatorname{Hom}}\left(C_{n-1}, G\right) \leftarrow \cdots,
$$

where $d_{n}^{*}(f)=f d_{n}$ for $f \in \operatorname{Hom}\left(C_{n-1}, G\right)$. We are interested in the $n$-dimensional quasi(co)homology module $\mathscr{H}^{n}(\operatorname{Hom}(C, G))$ of $\operatorname{Hom}(C, G)$. By Proposition 2.1 , we have the decompositions

and

$$
\operatorname{Hom}\left(\mathscr{H}_{n}(C), G\right)=\operatorname{Hom}\left(\underline{\mathscr{H}}_{n}(C), G\right) \oplus \operatorname{Hom}\left(\mathscr{H}_{n}(C), G\right)
$$

$$
\mathscr{H}^{n}(\operatorname{Hom}(C, G))=\underline{\mathscr{H}}^{n}(\operatorname{Hom}(C, G)) \oplus \overline{\mathscr{H}}^{n}(\operatorname{Hom}(C, G))
$$

Proposition 4.1. For any sequence $C$, for any module $G$ over the same ring and for any integer $n$, the mapping

$$
\alpha^{n}: \quad \mathscr{H}^{n}(\operatorname{Hom}(C, G)) \rightarrow \operatorname{Hom}\left(\mathscr{H}_{n}(C), G\right)
$$

defined as follows is a homomorphism: For

where

$$
f+\left(\operatorname{Im} d_{n}^{*} \cap \operatorname{Ker} d_{n+1}^{*}\right) \in \mathscr{H}^{n}(\operatorname{Hom}(C, G)),
$$

let

$$
f \in \operatorname{Im} d_{n}^{*}+\operatorname{Ker} d_{n+1}^{*} \subset \operatorname{Hom}\left(C_{n}, G\right),
$$

$$
\alpha^{n}\left(f+\left(\operatorname{Im} d_{n}^{*} \cap \operatorname{Ker} d_{n+1}^{*}\right)\right) \in \operatorname{Hom}\left(\mathscr{H}_{n}(C), G\right)
$$


be such that

where

$$
\alpha^{n}\left(f+\left(\operatorname{Im} d_{n}^{*} \cap \operatorname{Ker} d_{n+1}^{*}\right)\right): \quad c+\left(\operatorname{Ker} d_{n} \cap \operatorname{Im} d_{n+1}\right) \mapsto f(c),
$$

If

$$
c \in \operatorname{Ker} d_{n}+\operatorname{Im} d_{n+1} \subset C_{n} .
$$

and

$$
\underline{\alpha}^{n}=\alpha^{n} \mid \underline{\mathscr{H}}^{n}(\operatorname{Hom}(C, G))
$$

then

$$
\bar{\alpha}^{n}=\alpha^{n} \mid \overline{\mathscr{H}}^{n}(\operatorname{Hom}(C, G))
$$

and

$$
\underline{\alpha}^{n}: \underline{\mathscr{H}}^{n}(\operatorname{Hom}(C, G)) \rightarrow \operatorname{Hom}\left(\underline{\mathscr{H}}_{n}(C), G\right)
$$

$$
\bar{\alpha}^{n}: \quad \overline{\mathscr{H}}^{n}(\operatorname{Hom}(C, G)) \rightarrow \operatorname{Hom}\left(\overline{\mathscr{H}}_{n}(C), G\right)
$$

Proposition 4.2. For any sequence $C$, for any module $G$ over the same ring and for any integer $n$, if there exist isomorphisms $g_{n+1}, g_{n}$ and $g_{n-1}$ making

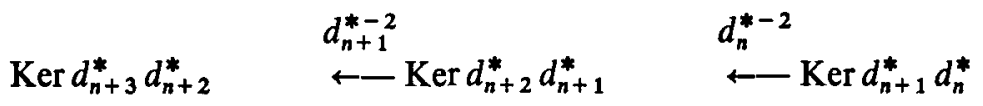

$$
\begin{aligned}
& \uparrow g_{n+1} \quad \uparrow g_{n} \quad \uparrow g_{n-1} \\
& \operatorname{Hom}\left(\operatorname{Ker} d_{n} d_{n+1}, G\right) \stackrel{d_{n+1}^{-2 *}}{\leftarrow} \operatorname{Hom}\left(\operatorname{Ker} d_{n-1} d_{n}, G\right) \stackrel{d_{n}^{-2 *}}{\longleftarrow} \operatorname{Hom}\left(\operatorname{Ker} d_{n-2} d_{n-1}, G\right)
\end{aligned}
$$

commutative, then

and

$$
\underline{\mathscr{H}}^{n}(\operatorname{Hom}(C, G))=H^{n}\left(\operatorname{Hom}\left(d^{-2} C, G\right)\right)
$$

$$
\operatorname{Hom}\left(\underline{H}_{n}(C), G\right)=\operatorname{Hom}\left(H_{n}\left(d^{-2} C\right), G\right) \text {. }
$$

Proof. Under the hypothesis, two semi-exact sequences $d^{*-2} \operatorname{Hom}(C, G)$ and Hom $\left(d^{-2} C, G\right)$ have the same $n$-dimensional homology module and Proposition 2.2 yields

$$
\underline{\mathscr{H}}^{n}(\operatorname{Hom}(C, G))=H^{n}\left(d^{*-2} \operatorname{Hom}(C, G)\right)=H^{n}\left(\operatorname{Hom}\left(d^{-2} C, G\right)\right)
$$

Needless to say, if $C$ is semi-exact, then the hypothesis of Proposition 4.2 is obviously satisfied. We cannot, however, make a statement which would be labelled as Proposition 4.3 corresponding to Proposition 3.3. Proposition 4.2 means that, under the hypothesis stated, the study of

$$
\underline{\alpha}^{n}: \quad \underline{\mathscr{H}}^{n}(\operatorname{Hom}(C, G)) \rightarrow \operatorname{Hom}\left(\underline{H}_{n}(C), G\right)
$$

is reduced to the study of the homomorphism

$$
H^{n}\left(\operatorname{Hom}\left(d^{-2} C, G\right)\right) \rightarrow \operatorname{Hom}\left(H_{n}\left(d^{-2} C\right), G\right)
$$

in the usual universal coefficient theorem for cohomology [1, p. 77]. In particular, we record

Proposition 4.4. For any sequence C of free modules over a principal ideal domain, for any 
injective module $G$ over the same domain and for any integer $n$, if the hypothesis of Proposition 4.2 is satisfied, then

defined by

$$
\alpha^{n}: \mathscr{\mathscr { H }}^{n}(\operatorname{Hom}(C, G)) \rightarrow \operatorname{Hom}\left(\mathscr{H}_{n}(C), G\right)
$$

$$
\underline{\alpha}^{n}\left(f+\left(\operatorname{Im} d_{n}^{*} \cap \operatorname{Ker} d_{n+1}^{*}\right)\right): \quad c+\left(\operatorname{Ker} d_{n} \cap \operatorname{Im} d_{n+1}\right) \mapsto f(c)
$$

is an isomorphism.

Since we do not have a statement which would be labelled as Proposition 4.3, we cannot make a statement which would be labelled as Corollary 4.5. The usual universal coefficient theorem for cohomology states among other things that, under certain circumstances, the homomorphism similar to our $\underline{\alpha}^{n}$ is an epimorphism. It is interesting to note that $\bar{\alpha}^{n}$ is a monomorphism. This is part of the following theorem.

THEOREM 4.6. Let $C$ be a sequence of modules and let $G$ be a module over the same ring. For any integer $n$, let

be a mapping defined by

$$
\bar{\alpha}^{n}: \quad \overline{\mathscr{H}}^{n}(\operatorname{Hom}(C, G)) \rightarrow \operatorname{Hom}\left(\overrightarrow{\mathscr{H}}_{n}(C), G\right)
$$

Then

$$
\bar{\alpha}^{n}\left(f+\left(\operatorname{Im} d_{n}^{*} \cap \operatorname{Ker} d_{n+1}^{*}\right)\right): \quad c+\left(\operatorname{Ker} d_{n} \cap \operatorname{Im} d_{n+1}\right) \mapsto f(c) .
$$

(1) $\bar{\alpha}^{n}$ is a monomorphism.

(2) If $C_{n-1}$ is projective, then

$$
0 \rightarrow \overline{\mathscr{H}}^{n}(\operatorname{Hom}(C, G)) \stackrel{\bar{\alpha}^{n}}{\rightarrow} \operatorname{Hom}\left(\mathscr{H}_{n}(C), G\right) \rightarrow \operatorname{Ext}\left(C_{n-1} / \overline{\mathscr{H}}_{n}(C), G\right) \rightarrow 0
$$

is exact.

(3) If $G$ is injective, then (regardless of whether $C_{n-1}$ is projective or not) $\bar{\alpha}^{n}$ is an isomorphism.

Proof. Let $j_{1}$ be the injection, i.e. the inclusion, of $\operatorname{Im} d_{n} d_{n+1}$ into $C_{n-1}$ and recall that $i_{2}=i_{2}(C)$ and $i_{1}=i_{1}(\operatorname{Hom}(C, G))$ are isomorphisms

and

$$
i_{2}(C): \overline{\mathscr{H}}_{n}(C) \nrightarrow \operatorname{Im} d_{n} d_{n+1}
$$

$$
i_{1}(\operatorname{Hom}(C, G)): \operatorname{Hom}\left(C_{n-1}, G\right) / \operatorname{Ker} d_{n+1}^{*} d_{n}^{*} \mathscr{\mathscr { H }}^{n}(\operatorname{Hom}(C, G))
$$

given in Proposition 2.3. From a short exact sequence

$$
0 \rightarrow \mathscr{\mathscr { H }}_{n}(C) \stackrel{j_{1} i_{2}}{\rightarrow} C_{n-1} \rightarrow C_{n-1} / \overline{\mathscr{H}}_{n}(C) \rightarrow 0,
$$

we obtain its fundamental exact sequence

$$
\begin{aligned}
& \cdots \leftarrow \operatorname{Ext}\left(C_{n-1}, G\right) \leftarrow \operatorname{Ext}\left(C_{n-1} / \overline{\mathscr{H}}_{n}(C), G\right) \\
& i_{2}^{*} j_{1}^{*} \\
& \leftarrow \operatorname{Hom}\left(\overline{\mathscr{H}}_{n}(C), G\right) \leftarrow \operatorname{Hom}\left(C_{n-1}, G\right) \leftarrow \operatorname{Hom}\left(C_{n-1} / \overline{\mathscr{H}}_{n}(C), G\right) \leftarrow 0 .
\end{aligned}
$$


A quick computation tells us that

$$
\operatorname{Ker} i_{2}^{*} j_{1}^{*}=\operatorname{Ker} d_{n+1}^{*} d_{n}^{*}
$$

In view of the commutative diagram

$$
\begin{array}{cl}
\operatorname{Hom}\left(\overline{\mathscr{H}}_{n}(C), G\right) & \stackrel{\mu}{\leftarrow} \operatorname{Hom}\left(C_{n-1}, G\right) / \operatorname{Ker} i_{2}^{*} j_{1}^{*} \\
\uparrow \bar{\alpha}^{n} & i_{1} \\
\overline{\mathscr{H}}^{n}(\operatorname{Hom}(C, G)) & \stackrel{H}{*} \operatorname{Hom}\left(C_{n-1}, G\right) / \operatorname{Ker} d_{n+1}^{*} d_{n}^{*},
\end{array}
$$

where $\mu$ is the monomorphism induced by $i_{2}^{*} j_{1}^{*}$ by factoring its domain, the fundamental exact sequence yields an exact sequence

$$
\because \leftarrow \operatorname{Ext}\left(C_{n-1}, G\right) \leftarrow \operatorname{Ext}\left(C_{n-1} / \overline{\mathscr{H}}_{n}(C), G\right) \leftarrow \operatorname{Hom}\left(\overline{\mathscr{H}}_{n}(C), G\right) \stackrel{\bar{\alpha}^{n}}{\leftarrow \overline{\mathscr{H}}^{n}}(\operatorname{Hom}(C, G)) \leftarrow 0
$$

The conclusions follow from this immediately.

Since we do not have a statement which would be labelled as Corollary 4.5 , we cannot make a statement which would be labelled as Corollary 4.7.

\section{REFERENCE}

1. S. MacLane, Homology (Berlin-Göttingen-Heidelberg, 1963).

EASTERN ILLINOIS UNIVERSITY

Charleston, Illinois 61920, U.S.A. 